\title{
VARIATION OF EVEN AND ODD PARITY IN THE SOLAR DYNAMO
}

\author{
A. BRANDENBURG ${ }^{1}$, R. MEINEL ${ }^{2}$, D. MOSS $^{3}$, I.TUOMINEN $^{1}$ \\ ${ }^{1}$ Observatory and Astrophysics Laboratory, University of Helsinki \\ Tähtitorninmäki, SF-00130 Helsinki, Finland \\ ${ }^{2}$ Sternwarte Babelsberg, Rosa-Luxemburg-Str. 17a, \\ 1591 Potsdam, German Democratic Republic \\ ${ }^{3}$ Dept. of Mathematics, The University, Manchester M13 9PL, England
}

\begin{abstract}
We have studied axisymmetric nonlinear $\alpha \omega$-dynamo models taking the interaction between even and odd parities fully into account. It turns out that the dominating type of symmetry is not always determined uniquely, but it can vary on a very long time scale compared to the period of the magnetic cycle. In some cases the frequency of this long term variation is close to the beat frequency of the two solutions with purely dipolar and purely quadrupolar parity. The occurrence of a second frequency is typical of solutions whose trajectory describes a torus in the phase space. We argue that this finding is of relevance for understanding secular variations observed in the Sun. For example measurements of sunspots indicate that the spot number on the northern hemisphere at present exceeds the number on the southern hemisphere. The reverse seems to have been the case at the end of last century.
\end{abstract}

\section{Introduction}

The magnetic field orientation in sunspot pairs is quite regular. The orientation reverses between the northern and southern hemisheres (odd parity) and alternates with the 22-year solar magnetic cycle (Hale \& Nicholson, 1925). Sunspot pairs are usually interpreted as an indicator for an azimuthal magnetic field in the solar convection zone. Flux ropes rise to the surface, because of magnetic buoyancy, where they can produce a pair of sunspots via the Parker (1979) mechanism.

Further, the radial component of the solar mean magnetic field $B_{r}$ shows a similar systematic variation to the azimuthal field. Stenflo \& Vogel (1986) and Stenflo (1988) showed that about $90 \%$ of the radial component of the magnetic field is of odd parity and oscillates with the 22 -year period. $10 \%$ of the $B_{r}$-field is of even parity and does not show the 22-year period but there are variations on a much shorter time scale.

Deviation of the solar magnetic field from a pure parity is of importance for understanding the solar dynamo. Traditional spherical mean-field dynamos (Steenbeck \& Krause, 1969; Krause \& Rädler, 1980) show pure parity magnetic field configurations when the dynamo number is close to the critical dynamo number $D_{\text {crit }}$. Only the solution corresponding to the first $D_{\text {crit }}$ is stable and therefore of relevance (Krause \& Meinel, 1988). 
Recently it has been demonstrated that for $\alpha \omega$-dynamos with weakly supercritical dynamo numbers $\left(D \approx 1.5 D_{\text {crit }}\right)$ the first pure solutions are unstable and only stable mixed parity solutions with contributions of even and odd parity are found (Brandenburg et al. 1989). The parity, measured by a parity parameter

$$
P=\left[E^{(\mathrm{S})}-E^{(\mathrm{A})}\right] /\left[E^{(\mathrm{S})}+E^{(\mathrm{A})}\right]
$$

can vary quasi-periodically on a time scale which is typically 3-30 times longer than the magnetic cycle period (Brandenburg, Moss \& Tuominen, 1989). $E^{(\mathrm{S})}$ and $E^{(\mathrm{A})}$ denote respectively the energies in the symmetric (even) and antisymmetric (odd) part of the magnetic field.

Oscillatory dynamos with mainly odd parity have been found which show, for approximately one third of the time, strong deviations from the odd parity, see Fig 1b. During such stages considerable contributions of even parity are present and the total magnetic field is weakened. This behavior has been tentatively associated with the phenomenon of Grand Minima (Brandenburg, Krause \& Tuominen, 1989). These models would predict an enhanced degree of "parity mixing" during a Grand Minimum.

These results are based on models, in which the $\alpha$-and $\omega$-effect operate in the entire sphere. However, these conditions are not met in the Sun. The purpose of the present paper is therefore to investigate more realistic models where the $\alpha$ - and $\omega$-effect are confined in a shell.

\section{Dynamos in a shell}

The $\alpha \omega$-dynamo can operate only in the convection zone and possibly in the thin overshoot layer below. Because of the very long diffusion time the radiative interior can be excluded from the computation of solar-type magnetic cycles. We do this by assuming a perfect conductor boundary condition at a radius $r=0.7 R$, where $R$ is the solar radius. We assume that the field continues as a potential field for $r>R$. Inside the computational domain $0.7 R<r<R$ we solve the dynamo equation (cf. Krause and Rädler, 1980)

$$
\partial B / \partial t=\operatorname{curl}(\boldsymbol{u} \times \boldsymbol{B}+\alpha \boldsymbol{B}-\eta \operatorname{curl} \boldsymbol{B})
$$

in spherical coordinates $(r, \theta, \phi)$ with $0 \leq \theta \leq \pi$, restricting ourselves, however, to axisymmetry (i.e. $\partial / \partial \phi=0$ ). Inside the shell we take $u=\hat{\phi} \Omega r \sin \theta$ with $\partial \Omega / \partial r=C_{\omega} \eta / R^{3}=$ const and $C_{\omega}=-1000$. We assume that $\alpha$ depends on $B$ via $\alpha=C_{\alpha} \cos \theta(\eta / R)(1+$ $\left.B^{2} / B_{0}^{2}\right)^{-1}$ with $B_{0}=\sqrt{\mu \rho} \eta / R$. We find the critical dynamo numbers for $D_{\text {crit }}^{(\text {odd }}=-5620$ and $D_{\text {crit }}^{(\text {even }}=-5570$ for even and odd parity, where $D=C_{\alpha} C_{\omega}$. Note that, in contrast to dynamos in a complete sphere, the S-type (even) solution bifurcates first from the trivial solution. This feature has been found already by Roberts (1972). (We have investigated here only the case $D<0$, because only then the field belts migrate toward the equator.)

The minimum and maximum energies and frequencies for both solutions are listed in the Table 1. Note that these values for both parities lie very close together. Near to the first bifurcation $(D=-5570)$ the S-type solution is stable. For $D=-8000 \ldots-10000$ only the A-type solution is stable. These results were obtained with a numerical resolution of $41 \times 81$ mesh points. For a lower resolution of only $21 \times 41$ mesh points we find the mixed parity 
solution still at $D=-10^{4}$ (this solution is shown in Fig. 1a). Since the bifurcation points are shifted slightly as the resolution is increased we expect that, for a higher resolution, this mixed parity solution corresponds to $D \approx-6000 \ldots-8000$.

Table 1: Energies and frequencies for even and odd parity solutions of the $\alpha \omega$-dynamo in a shell for $C_{\omega}=-10^{3}$

\begin{tabular}{|c|ccc|ccc|ccc|}
\hline & \multicolumn{3}{|c|}{$E_{\min }$} & \multicolumn{3}{c|}{$E_{\max }$} & \multicolumn{3}{c|}{ frequency } \\
\hline$C_{\alpha}$ & 6 & 8 & 10 & 6 & 8 & 10 & 6 & 8 & 10 \\
\hline $\mathrm{A}$ & 0.040 & 0.279 & 0.503 & 0.054 & 0.339 & 0.590 & 138.6 & 150.3 & 158.7 \\
$\mathrm{~S}$ & 0.043 & 0.277 & 0.499 & 0.059 & 0.344 & 0.601 & 138.1 & 149.6 & 160.3 \\
\hline
\end{tabular}

The temporal variation of $E$ and $P$ for a mixed solution is shown in the figure below (left panel). ( $D=-10^{4}$ with $21 \times 41$ mesh points.) For comparison we have included in the right panel the solution for the entire sphere for $D=-8500$ and $41 \times 81$ mesh points (Brandenburg, Krause \& Tuominen, 1989).
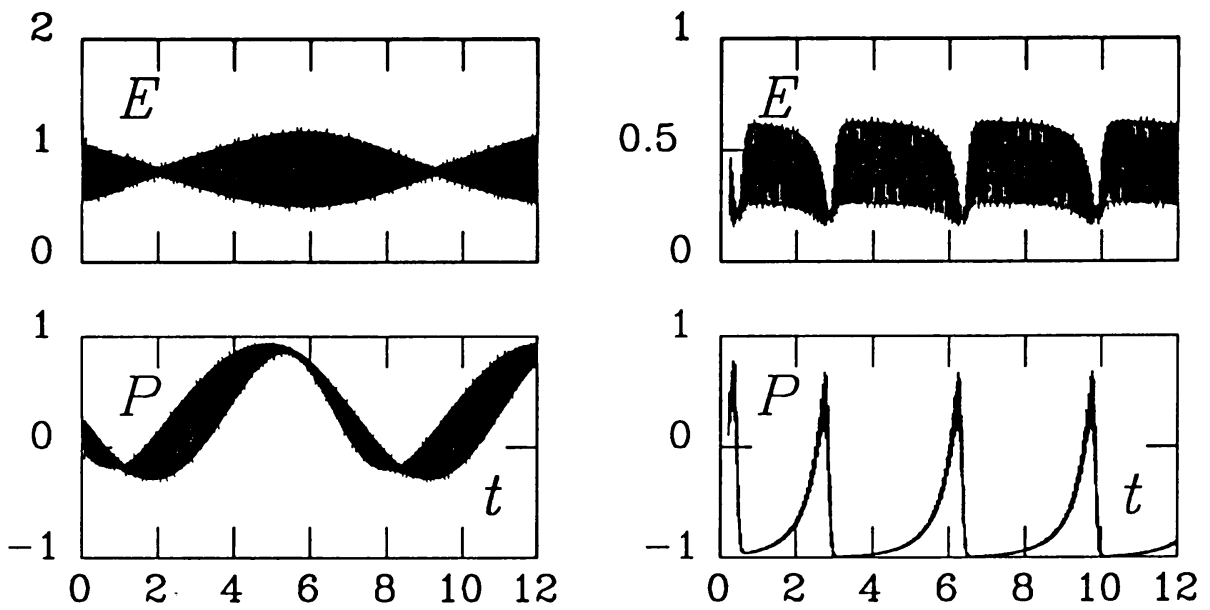

Fig. 1. Left panel: Evolution of energy and parity parameter for the shell dynamo and $C_{\alpha}=10$. Right panel: Same, but for a dynamo operating in the entire sphere

The period of the mixed solutions is very long (about 100 times larger) compared to the period of the basic magnetic cycle. The long term period is in this case close to the beat frequency between the two pure (unstable) solutions. The beat frequency is in this case very low, because the frequencies of the pure solutions lie very close together.

Neglecting the fact that solar Grand Minima occur irregularly (this has been modelled by Belvedere and Proctor (1989) with a highly supercritical one dimensional dynamo) it seems that their recurrence time is not much longer than 10-30 magnetic cycle periods. It would be therefore of interest to look for models with a period ratio smaller then 100 , which is the value found for shell dynamos with radially constant $\alpha$-and $\omega$-profiles. 


\section{Discussion and conclusions}

The shell dynamos discussed in this paper display a somewhat different behaviour to dynamos operating in the entire sphere. The critical dynamo numbers for even and odd parity lie very close together and also the difference in the magnetic cycle periods are so small that the corresponding beat frequency of the mixed mode solution is smaller by a factor 100 than the magnetic cycle frequency.

Previous conclusions concerning the connection to Grand Minima have to be reconsidered in the case where the dynamo is confined to the outer envelope. It is also very important that it is now an oscillatory even parity mode which becomes first excited. This seems somewhat similar to the 1-D model by Jennings and Weiss (1989), which also shows an even mode being excited first. This is however a stationary one.

The shell dynamos have the property that the fields in the northern and southern hemisphere are quite separated, which is not the case for dynamos operating in a full sphere where fields can extend down to the center. This is supported also by the very similar field geometry found for even and odd parity solutions (apart from the opposite sign in either hemisphere).

Of course we must be aware of the high degree of simplifications made in these models. These simplifications may account for some important qualitative discrepancies between our models and the observations. For example, Grand Minima appear irregularly rather than quasi-periodically. It is likely that such features occur once the fields are dynamically coupled to the differential rotation. Also the even parity contributions to the solar field do not show any periodic variations with approximately the 22-year cycle period, whereas in our models fields of both parities oscillate with a common frequency.

Acknowledgements. We thank F. Krause and G. Rüdiger for many interesting discussions.

\section{References}

Belvedere, G., Proctor, M. R. E.: 1989, these proceedings

Brandenburg, A., Krause, F., Meinel, R., Moss, D., and Tuominen, I.: 1989, Astron.

Astrophys. 213, 411

Brandenburg, A., Moss, D., Tuominen, I., 1989, Geophys. Astrophys. Fluid Dyn. (in press)

Brandenburg, A., Krause, F., Tuominen, I.: 1989, in Turbulence and Nonlinear Dynamics

in MHD Flows, eds. M. Meneguzzi, A. Pouquet and P. L. Sulem, Elsevier Science

Publ. B.V. (North-Holland), p. 35-40

Jennings, R., Weiss, N. O.: 1989, these proceedings

Hale, G. E., Nicholson, S. B. : 1925, Astrophys. J. 62, 270

Krause, F., Rädler, K.-H.: 1980, Mean-Field Magnetohydrodynamics and Dynamo Theory, Akademie-Verlag, Berlin

Krause, F., Meinel, R. : 1988, Geophys. Astrophys. Fluid Dyn. 43, 95

Parker, E. N.: 1979, Cosmical magnetic fields, Clarendon press, Oxford

Roberts, P. H.: 1972, Phil. Trans. Roy. Soc. 274, 663

Steenbeck, M. and Krause, F.: 1969, Astron. Nachr. 291, 49.

Stenflo, J.O., Vogel, M. : 1986, Nature 319, 285

Stenflo, J.O. : 1988, Astrophys. Spa. Sci. 144, 321 